\title{
Comparative Simulations for SARA and AODV Routing Algorithms in Ad hoc Network using NS2
}

\author{
Tamer Hashem Farag \\ Management Information Systems Department \\ College of Applied Studies and Community Services \\ University of Dammam
}

\author{
Reem Farag Ahmed \\ Department of Mathematics \\ Faculty of Science \\ Cairo university
}

\begin{abstract}
The past two decades noticed a great development in ad hoc wireless networks. Although most of the routing algorithms that have been produced to ad hoc networks find the shortest discovered path, they have different network performances. It is hard to predict which routing algorithm will perform well under different scenarios. In this paper, an empirical study of the elements that may affect a routing algorithm's performance using NS2 simulator is presented. AODV and SARA routing algorithms have been chosen to apply the study on and their performances have been investigated under different scenarios. NS2 has been used to simulate three topologies and their performances have been measured. According to the results, it is suggested to use AODV routing algorithm in low interference scenarios and to use SARA routing algorithm in high interference scenarios.
\end{abstract}

\section{General Terms}

Routing algorithms, IEEE 802.11 standards, Simulation

\section{Keywords}

Ad hoc Wireless Network,Ad hoc Routing Algorithms, Simple Ant Routing Algorithm (SARA), On-demand Algorithms, Ad hoc Ondemand Distance Vector (AODV), IEEE 802.11a/b/g

\section{INTRODUCTION}

An ad hoc wireless network is a collection of nodes that communicate through a wireless medium. Each node can move anytime anywhere which may cause a topology change. The transmission range is usually limited in ad hoc wireless network, so packets may need several intermediate nodes to be delivered. Therefore, each node in ad hoc wireless network acts as a router and a node at the same time. Ad hoc wireless networks have no fixed infrastructure and usually do not use any central administration. Therefore, this type of networks is useful in disasters and rescue situations. Designing a routing algorithm for ad hoc wireless networks is a tough operation because ad hoc wireless networks are self-organized, selfconfigured, have no fixed infrastructure and their topologies may change [1][2].

There are a lot of routing algorithms that introduced for ad hoc networks. These routing algorithms can be divided into two categories: proactive and reactive routing algorithms. In proactive rout- ing algorithms (also known as Table-Driven routing algorithms), routes are maintained for the whole topology and they are periodically updated. Examples of such routing algorithms are DSDV [3] and OLSR [4]. In reactive routing algorithms (also known as On-Demand routing algorithms) routes are created when they are needed only. These routing algorithms may reduce the network overhead compared with proactive routing algorithms, because there are no periodic updates. Examples of such routing algorithms are AODV [5] and DSR [6][1][7].

Any routing algorithm finds the best route according to a specific criteria : shortest path, minimum delay and minimum overhead, and it is proactive routing algorithm. The find route process is an optimization problem. which can be solved through heuristic algorithms. Swarm Intelligence algorithms is a class of heuristic algorithms.

Swarm Intelligence algorithms are inspired from nature. Their basic idea based on the collective behavior. One of the Swarm Intelligence algorithms is Ant Colony Optimization (ACO) algorithms which simulates the ants colony behavior. An ant colony is selforganized, all colony members are cooperated to finish specific jobs. Ants try to find the shortest discovered path from its nest to the food source by following the trials of the deposition of a chemical material called pheromone. Routing algorithms that depend on the idea of ACO technique are called ant routing algorithms. In such algorithms, there are control packets (ants) used to collect information about the network to find the shortest discovered path. Examples of such routing algorithms are ARA [8] and SARA [9]|2]|[10]. By simulation of the routing algorithms we can measure their performances. One of the most popular simulators is the NS2. NS2 is used to simulate the routing algorithms, application layer protocols, transport layer protocols and others. The most important step in simulating the ad hoc wireless network is to build the network connections. The connection setup is determined by both of Media Access Control (MAC) and physical layer specifications.

The Institute of Electrical and Electronics Engineers (IEEE) developed a wireless network standard called IEEE 802.11 in 1991. The first published standard was in 1997. IEEE 802.11 standard contains a set of MAC and physical layer specifications for the wireless network. IEEE 802.11 standard has many advanced versions like IEEE 802.11b, IEEE 802.11a, IEEE 802.11g and IEEE 802.11n standards. They are different in the frequency band and the specifications [11][12]. Here we will simulate the routing algorithms with different wireless network signals standards. 


\section{RELATED WORK}

There are many comparisons studies between the routing algorithms that have been introduced. In this section, some of these comparisons will be listed.

Samir R.Das and et al. in [7] made a comparison between several ad hoc routing algorithms. Those algorithms divided into proactive and reactive algorithms. The results show that proactive algorithms have better delay and packet delivery than the reactive algorithms and reactive algorithms have lower routing overhead than the proactive algorithms.

Iliya Enchev Pervasive and et al. in [15] produced a survey for ant routing algorithms. It contains brief descriptions for ARA, SARA, ANSI, AntHocNet, HOPNET and bee-inspired routing algorithms. They did not make any new results only show the original ones. Dilpreet Kaur and Naresh Kumar in [17] introduced performance comparisons between AODV, OLSR, DSR and DSDV ad hoc routing algorithms. The performance metrics used in the comparisons are packet delivery ratio, average delay, normalized routing load, throughput and jitter. The performances have been measured under two levels of mobility and two levels of network traffic. The results show that AODV has the best throughput performance under low traffic while DSDV has the best throughput performance under high traffic. In high traffic, the throughput performances of OLSR, DSR and DSDV operate good in despite of the performances AODV and TORA. DSDV has the worst normalized routing load performance while TORA has the best normalized routing load performance. OLSR and DSDV produce bad performances in both jitter and the average delay.

Tarunpreet Bhatia and A.K. Verma in [18] produced performance comparisons between the ad hoc proactive, reactive and hybrid routing algorithms. The routing algorithms which have used to be analyzed are DSDV, OSLR, AODV, DSR and ZRP. The performance metrics that have evaluated are throughput, packet delivery fraction, normalized routing load and average end to end delay. The performances have measured under different network size, node speed and pause time. The results show that AODV has the best performances while ZRP has the worst performances among the five routing algorithms. The performances of reactive routing algorithms beat the performances of proactive routing algorithms under the metrics of throughput and packet delivery fraction. The performances of proactive routing algorithms are better than the other algorithms under the metrics of average end to end delay and normalized routing load.

Merin Skariah and Prof.Dr. C.D. Suriyakala in [11] made a network performance comparison between DSDV, AODV and DSR routing algorithms under the specifications of IEEE $802.11 \mathrm{~b}$ standard. The results show that AODV has the best network performance in throughput and packet loss. Results also show that DSDV has the worst results in throughput, and DSR has the best results in end-toend delay.

Cristian Hernandez Benet and et al. in [12] made a genetic algorithm for forecasting the TCP available bandwidth and made a study about the impact of the primary users on the TCP available bandwidth using IEEE $802.11 \mathrm{~g}$ standard. The results show that the TCP available bandwidth increases when the ideal intervals are longer.

\section{BACKGROUND}

An empirical study of the elements that may affect a routing algorithm's performance is presented in this paper. The study has been applied on two on demand algorithms: one known by its sta- bility (AODV) and another one known by its ability to reduce the network overhead (SARA). The IEEE 802.11a, IEEE 802.11b and IEEE $802.11 \mathrm{~g}$ standards have been implemented through simulator on these algorithms, and that is applied with data rates 24,11 and 54 Mbps respectively. Then, network performance comparisons have been made between them under different elements and their behaviors have been analyzed under these elements. A Similar comparison has been made between them in [9], the results show that SARA has better throughput performance than AODV under the specification of IEEE $802.11 \mathrm{~b}$ with data rate equals to $2 \mathrm{Mbps}$. Descriptions of both AODV and SARA routing algorithms, IEEE 802.11, IEEE 802.11b, IEEE 802.11a and IEEE $802.11 \mathrm{~g}$ standards will be provided in the next subsections.

\subsection{AODV routing algorithm}

AODV routing algorithm is an On-Demand algorithm and is an enhancement version of DSDV routing algorithm. AODV algorithm consists of two phases: path discovery and path maintenance. Path discovery phase starts when a source node wishes to send data to a destination node, if a source node has a route to the destination node in its routing table, the source node will start to send the data, if not the source node will broadcast a request (RREQ) packet to its neighbors, a node that receives the RREQ for first time will update its routing table and will rebroadcast the message to its own neighbors. Any RREQ packet is uniquely identified by source address and broadcast id. A node can receive many copies of the same RREQ packet so, it drops any copy of the received RREQ and never rebroadcast it. Only a destination node or a node has a valid route to the destination node can reply by a reply (RREP) packet to the source node. When a RREP packet received by the source node, the path establishes and starts the data sending. Path maintenance takes place; if a source node moved, a new path discovery initiates. If the destination node or an intermediate node belongs to the route moved, an error (RERR) packet sends to the source node and any node receives this error packet updates its routing table by setting the number of hops to $\infty$, when the RERR packet received by the source node, a new path discovery initiates [5].

\subsection{SARA routing algorithm}

SARA routing algorithm is an On-Demand algorithm and is an enhancement version of ARA routing algorithm. SARA routing algorithm consists of three phases: route discovery, route maintenance and route repair. Route discovery phase starts when a source node wishes to send data to a destination node, if the source node has a route to the destination node, it will use it and will start data transmission. If not, a source node sends a FANT packet to its neighbors but only one of them will rebroadcast the FANT packet. A node that receives the FANT packet updates its routing table to the source node. Only the destination node or the node that is responsible of forwarding the FANT are capable of replying by a BANT packet. BANT packet will be sending to the source node. Any node receives a BANT packet, updates its routing table to the destination node. Within a certain period if the source node does not receive any BANT packet, another FANT will be sent. Upon the reception of the first BANT the route is established and the data is sent. Route maintenance takes place with the reception of any packet, the opposite direction of the link where the packet came from, will be marked which means the pheromone value of this link will be increased by a constant value. By time, the evaporation will be represented by decreasing the pheromone each constant period by constant value. Route repair will start when a link failure detected. SARA algorithm uses a search for alternative route within 
Table 1. IEEE 802.11 specifications

\begin{tabular}{|c||c|c|c|}
\hline & IEEE 802.11b & IEEE 802.11a & IEEE 802.11g \\
\hline \hline Slot time & $20 \mu \mathrm{s}$ & $9 \mu \mathrm{s}$ & $9 \mu \mathrm{s}$ \\
\hline CWMin & 31 & 15 & 15 \\
\hline CWMax & 1023 & 1023 & 1023 \\
\hline SIFS & $10 \mu \mathrm{s}$ & $16 \mu \mathrm{s}$ & $16 \mu \mathrm{s}$ \\
\hline DIFS & $50 \mu \mathrm{s}$ & $34 \mu \mathrm{s}$ & $34 \mu \mathrm{s}$ \\
\hline Bandwidth & $22 \mathrm{Mbps}$ & $20 \mathrm{Mbps}$ & $20 \mathrm{Mbps}$ \\
\hline Data rate & $11 \mathrm{Mbps}$ & $24 \mathrm{Mbps}$ & $54 \mathrm{Mbps}$ \\
\hline Preamble length & $144 \mathrm{bits}$ & $96 \mathrm{bits}$ & $96 \mathrm{bits}$ \\
\hline PLCPHeaderLength & $48 \mathrm{bits}$ & $40 \mathrm{bits}$ & $40 \mathrm{bits}$ \\
\hline Basic Rate & $1 \mathrm{Mbps}$ & $6 \mathrm{Mbps}$ & $6 \mathrm{Mbps}$ \\
\hline PLCPDataRate & $1 \mathrm{Mbps}$ & $6 \mathrm{Mbps}$ & $6 \mathrm{Mbps}$ \\
\hline Frequency & $2.4 \mathrm{GHz}$ & $5.18 \mathrm{GHz}$ & $2.4 \mathrm{GHz}$ \\
\hline
\end{tabular}

a ring with $\mathrm{TTL}=2$, if this repair fails to find a solution, a new route discovery phase will be initiated [9].

\subsection{IEEE 802.11}

MAC is established based on the Carrier Sense Multiple Access with Collision Avoidance (CSMA/CA) mechanism. CSMA/CA is based on the backoff algorithm where any node wants to send a packet; first it senses the medium, if it busy, the node defers its transmission until the medium is idle. If the medium is idle, the node senses the medium for an interval, then the node picks up a random backoff interval and decreases it as long as the medium is idle [11].

NS2 simulator contains parameters that represent the MAC and physical layer specifications of an IEEE 802.11 standard. One of the MAC parameters in NS2 is a special mechanism called RTS/CTS (concerns by the collision), where the sender first sends an RTS message and the destination replies with a CTS message. After that the actual DATA/ACK transmission occurs. You can control either to open or close the RTS/CTS mechanism in NS2. The mechanisms of both CSMA/CA and RTS/CTS illustrated in Fig.1.

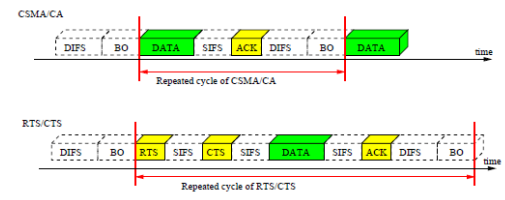

Fig. 1. CSMA/CA and RTS/CTS diagram

IEEE 802.11 standard has many advanced versions like IEEE 802.11b, IEEE 802.11a and IEEE $802.11 \mathrm{~g}$ standards. They are different in MAC and physical layer specifications. In table 1, the specifications of the IEEE 802.11b, IEEE 802.11a and IEEE $802.11 \mathrm{~g}$ standards that have been used in the simulation scenarios.

\section{PROBLEM FORMULATION}

In the empirical study that have been made, elements that can affect the routing algorithm's performance have been chosen then they have been thoroughly investigated. The candidate elements were found to be: different interference levels, different IEEE 802.11 specifications and different data rates. To practically apply this study, NS2 simulator has been used to measure the performances of three topologies represent three levels of interference. Also, the three topologies represent three levels of mobility due to the effect of the interference. If there are many nodes sending data hence there is interference between them the nodes affected by the interference can be considered as they moved from their locations. Therefore, the interference effect can be considered as it affects the mobility and existence of a node in an ad hoc wireless network. Consider the situation of many nodes two of them are sending data the other nodes that are not belong to the active path can be considered as dead nodes or can be considered as they have left the ad hoc wireless network so, this situation also represents the mobility in another way. From previous, the mobility effect can be accomplished by many ways rather than moving the nodes. It has been assumed that the maximum transmission range is $100 \mathrm{~m}$ between any two nodes. In the following subsections, a detailed description of the simulated networks' structures will be given.

\subsection{First Topology}

The topology area is $100 \times 50 \mathrm{~m}^{2}$. This topology consists of fifty six nodes distributed in seven rows. Each row has eight nodes as illustrated in Fig.2. The horizontal distance between any two adjacent nodes is ten meters, and the vertical distance between any two adjacent nodes is five meters. The distance between the two nodes located in the extreme opposites is seventy six meters. This topology represents the highest level of interference among our proposed topologies due to the high density of nodes in a small area. This topology represents the highest level of mobility.

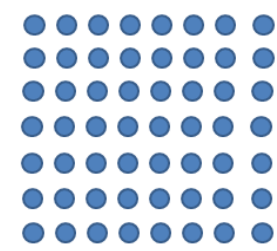

Fig. 2. First topology diagram

\subsection{Second Topology}

The area of this topology is $345 \times 300 \mathrm{~m}^{2}$. This topology also consists of fifty six nodes distributed in seven rows. Each row has eight nodes and the distance between any two adjacent nodes is fixed. The vertical distance between any two adjacent nodes is equals to the horizontal distance between any two adjacent nodes and is forty five meters. The distance between any two adjacent nodes is nearly the half of the maximum transmission range so this topology represents the intermediate level of interference among our proposed topologies. This topology represents the intermediate level of mobility.

\subsection{Third Topology}

The topology area is $800 \times 800 \mathrm{~m}^{2}$. This topology consists of forty one nodes distributed in six stars. Each star consists of six nodes, one is placed at the center and the other five nodes are placed around the center as illustrated in Fig.3. Each node in the star is connected only with the center node, which means that the nodes are placed out of range of each other. This situation is happened because the outer star nodes are located with seventy two degrees from the center node, and are located with approximately ninety meters distance from the center. Only in the central star, the outer 
nodes are located with approximately one hundred meters. Each star is placed out of range of the other stars, so there is no interference between any two stars. There are five nodes located to connect between the stars and the central star. For any transmission, there is only one path to follow. Therefore, this topology represents the lowest level of interference among our proposed topologies. This topology represents the lowest (zero) level of mobility.

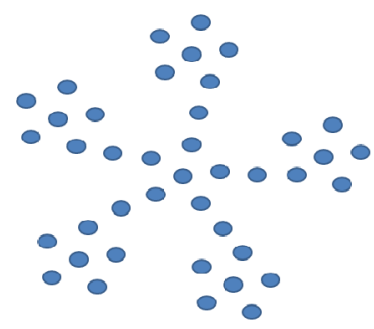

Fig. 3. Third topology diagram

\section{SIMULATION RESULTS}

In this section, all the simulation results will be shown. Three topologies have been used in the simulations to represent different levels of interference. In each topology, comparisons have been made under different IEEE 802.11 specifications. All the simulation results have been executed on NS2.31 simulator which has been installed on Ubuntu 10.04 operating system. The AODV builtin code that exists in NS2 simulator has been used and the latest version of the SARA code that is available on [?] has been used. NS2 does not have ready models for the IEEE 802.11 standards. The IEEE 802.11a, IEEE 802.11b and IEEE 802.11g standards have been built and have been used in the simulation scenarios. TCP and UDP algorithms have been used as the transport layer algorithms in the simulation results. FTP and CBR applications have been used as the application layer's applications. The average throughput and end-to-end delay are the performance metrics that have been used. In the next sections, the results that have been achieved from the three topologies will be shown.

\subsection{Mobility-Interference}

This group of results gives an evident that the interference has the same effect of the mobility. The simulation scenario contains two parallel connections interfere each other. One of the connection pairs moves away which enhances the metric performances of the routing algorithm under the three standards as illustrates in Fig.4.

\subsection{First Topology}

In this set of simulation results, each simulation run is executed for 50 seconds. This set of simulation results consists of four different scenarios. Each scenario consists of five sequential connections. FTP and CBR applications are used to transfer the data with packet sizes 512 and 1000 bytes respectively. Each scenario is repeated for ten times for FTP and is repeated for fifteen times for CBR. Due to the high density of nodes, multiple paths are available which reduces the interference effect. The two routing algorithms have the same behavior for both performance metrics under the three IEEE 802.11 standards with a privilege for AODV routing algorithm with

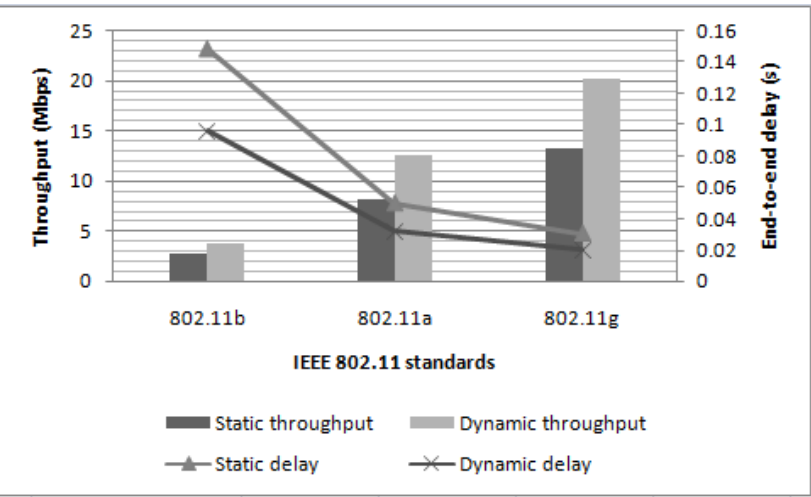

Fig. 4. The performances of SARA algorithm

CBR as illustrates in Fig.5 and Fig.6. In Fig.5 and Fig.6, the endto-end delay decreases while the data rate increases.

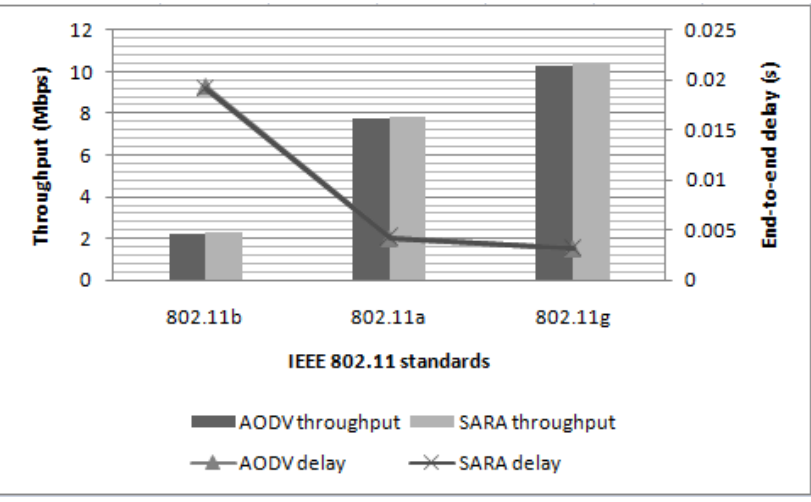

Fig. 5. First topology: Performances using FTP

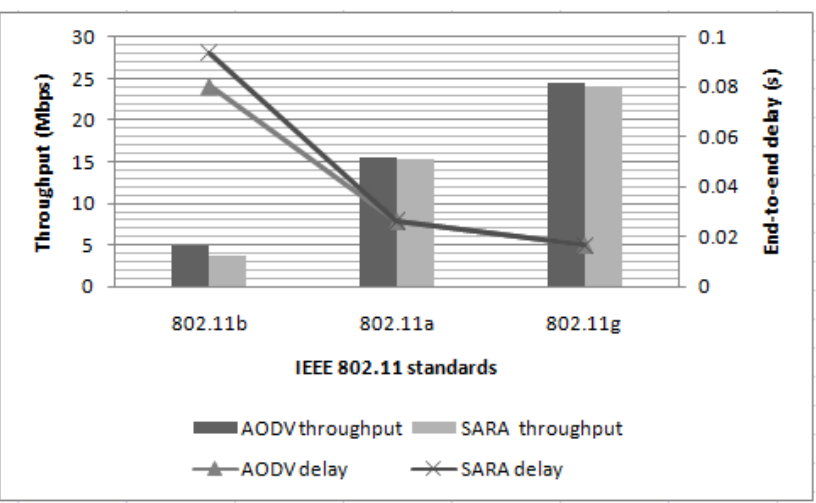

Fig. 6. First topology: Performances using CBR 


\subsection{Second Topology}

In this set of results, each simulation run is executed for 50 seconds. Each simulation run consists of five sequential connections. Four scenarios are used for simulations. The two applications FTP and CBR are used to transfer the data with packet sizes 512 and 1000 bytes respectively. Each scenario is repeated for ten times when using FTP and is repeated for fifteen times when using CBR. The interference affects the performance of AODV routing algorithm unlike the performance of SARA routing algorithm which deals better with the interference effect. The results show that SARA has better performances than AODV for both performance metrics under the three IEEE 802.11 standards when FTP is used as illustrates in Fig.7. When CBR is used, the throughput performance of SARA is better than the performance of AODV under the three standards and the two algorithms have the same end-to-end delay performance behavior as illustrates in Fig.8.

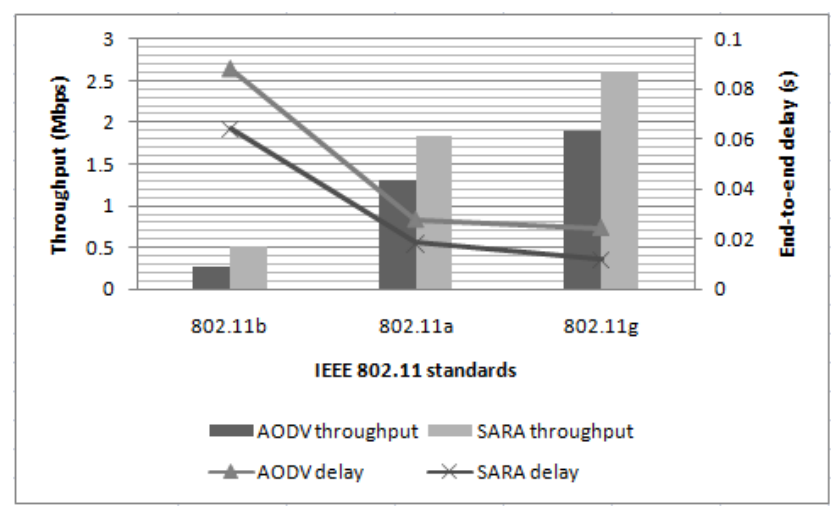

Fig. 7. Second topology: Performances using FTP

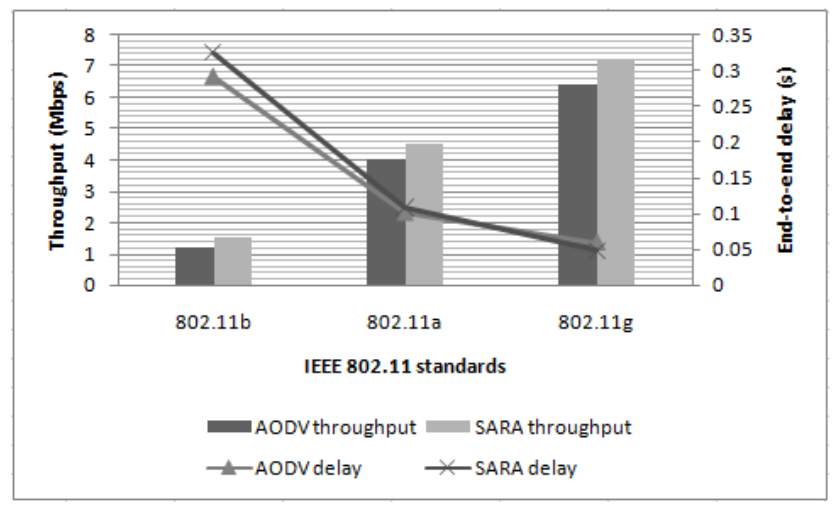

Fig. 8. Second topology: Performances using CBR

\subsection{Third Topology}

In this set of results, each simulation run is executed for 40 seconds and is repeated for fifteen times. Two applications are used for transferring the data: CBR with a packet size 1000 bytes and FTP with a packet size 512 bytes. Three sets of scenarios have been studied. There are four types of nodes in this topology; center node which represents the core of the star, deep node which represents the outer node of the star, first node which represents the node that connects the star with the connector node and before node which represents the connector node. Two network performances have been made.

5.4.1 The First Set. In this set of simulation results, a set of 32 different scenarios is used; two different scenarios represent each type of the 16 possible node combinations. Each scenario consists of two sequential connections. The results show that the two algorithms performances are so close to each other under $802.11 \mathrm{~b}$ standard while the metric performances of SARA are better than the metric performances of AODV under 802.11a and 802.11g standards. The throughput performance of SARA algorithm is better than the throughput performance of AODV routing algorithm under the three standards when the CBR is used as illustrates in Fig.9, while the throughput performance of AODV is better than the performance of SARA under $802.11 \mathrm{~b}$ standard when the FTP is used as illustrates in Fig.10. The end-to-end delay performance of SARA algorithm is better than the performance of AODV under the three standards when FTP is used as illustrates in Fig.10, while the performance of AODV is better than the performance of SARA under 802.11b standard when CBR is used as illustrates in Fig.9.

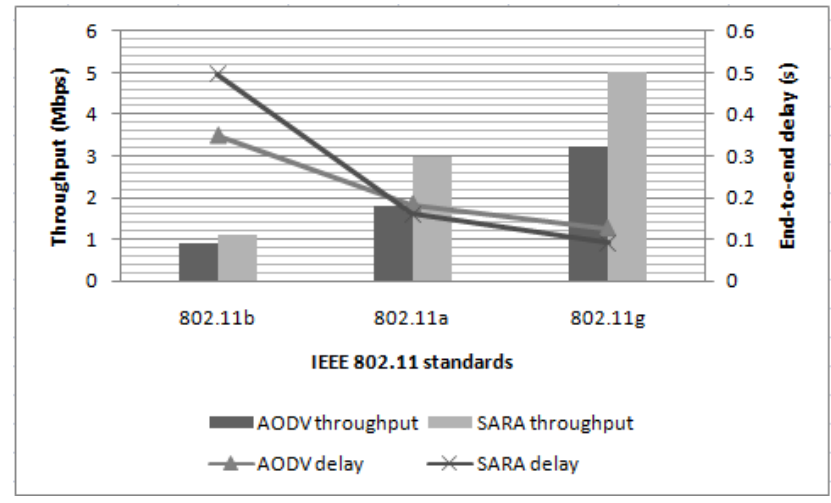

Fig. 9. First set - Performances using CBR

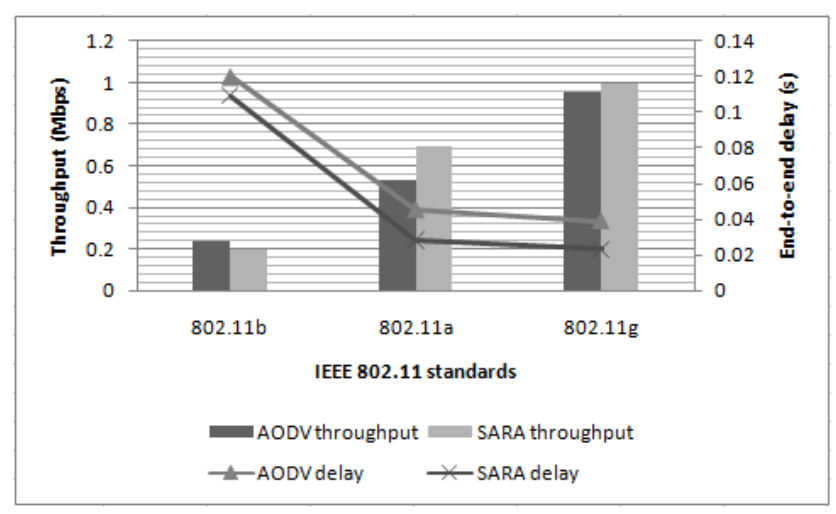

Fig. 10. First set - Performances using FTP 
5.4.2 The Second Set. In this set of simulation results, a set of 48 different scenarios is used; three different scenarios represent each type of the 16 possible node combinations. Each scenario consists of one connection. The results show that the throughput performance of SARA algorithm is better than the performance of AODV algorithm under the three standards when either CBR or FTP is used as illustrates in Fig.11 and Fig.12. The end-to-end delay performance of SARA algorithm is better than the performance of AODV under the three standards when FTP is used as illustrates in Fig.12, while the performance of AODV is better than the performance of SARA when CBR is used as illustrates in Fig.11.

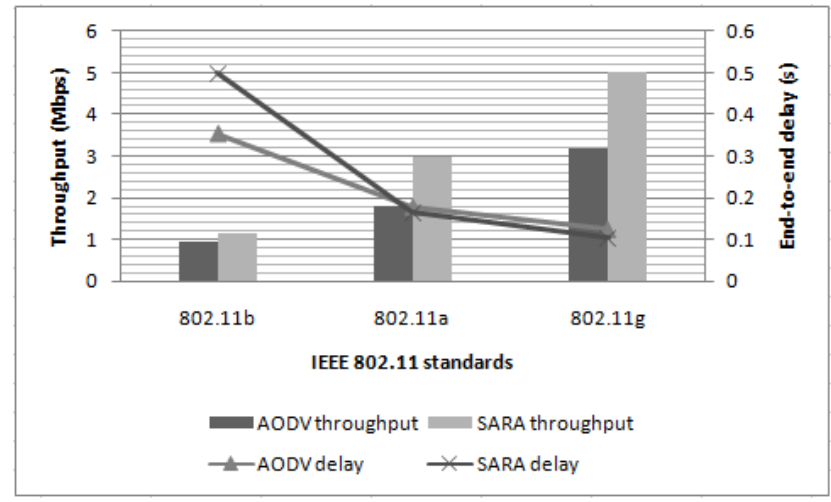

Fig. 11. Second set - Performances using CBR

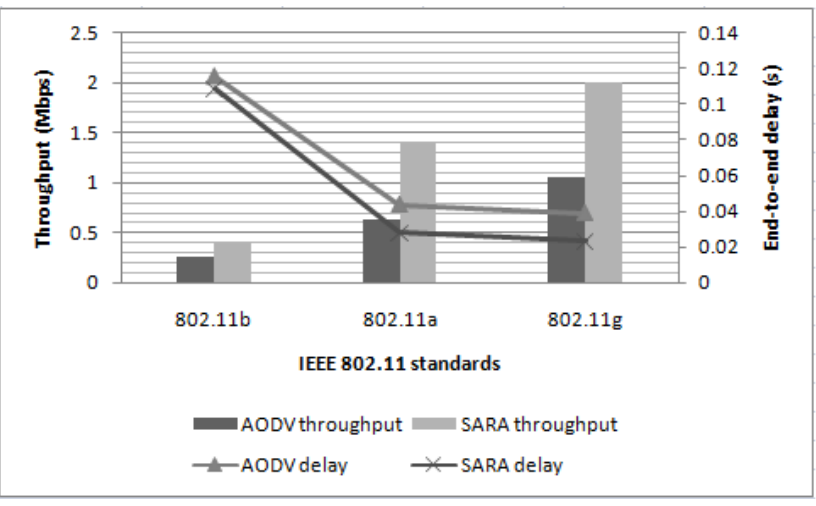

Fig. 12. Second set - Performances using FTP

\subsection{Opened/Closed RTS/CTS Mechanism}

In this set of simulation results, a set of 211 different scenarios is used, each scenario contains one connection. Source and destination nodes are selected to cover all types of nodes in this topology, so we have sixteen possible combinations. This set of results examine the effect of opening and closing the RTS/CTS mechanism on the two routing algorithms under the three standards. The FTP application has been used in this set of results. Opening the RTS/CTS does not affect the results, SARA routing algorithm has a better performance than AODV routing algorithm under the three standards for both metrics as illustrates in Fig.13 and Fig.14.

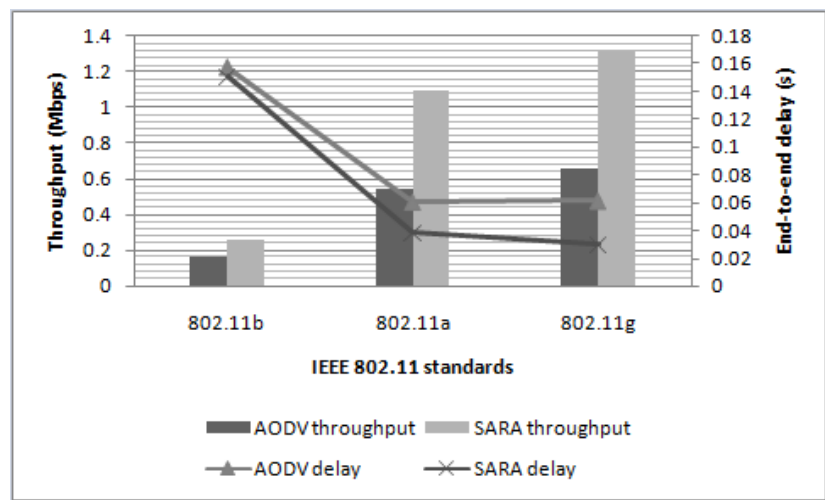

Fig. 13. Third set - Performances with Opened RTS/CTS

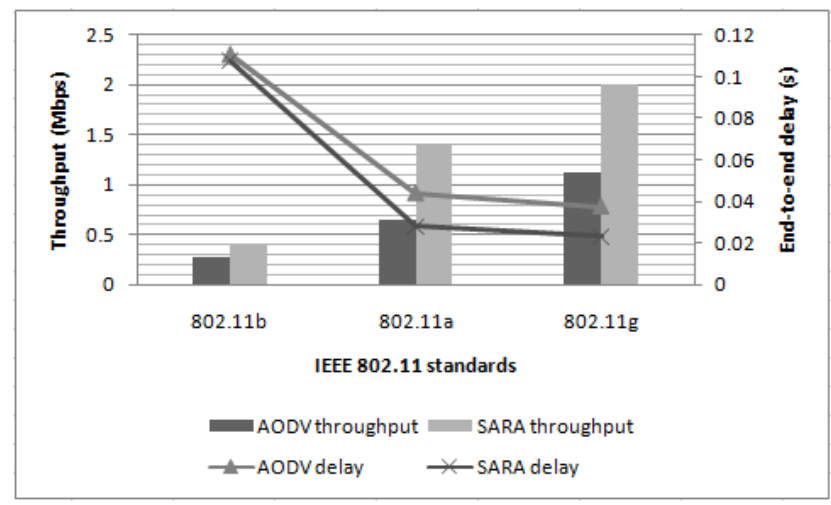

Fig. 14. Third set - Performances with closed RTS/CTS

\subsection{Two Parallel Connections}

This set of simulation results is obtained by simulating two parallel connections. The simulation run is executed for 40 seconds. CBR has used to transfer the data with packet size of 1000 bytes. With the existence of two parallel connections, the interference's effect shows in the three topologies and the performance of AODV algorithm enhances with the decrement of the interference. In the first topology, the throughput performance of SARA is better than the performance of AODV under the three standards while the end-toend delay performance of AODV is better than the performance of SARA under $802.11 \mathrm{~b}$ as illustrates in Fig.15. In the second topology, AODV has better performance than SARA for both metrics under the three standards as illustrates in Fig.16. In the third topology, the performance of AODV is better than SARA for both metrics under the three standards except the throughput performance under 802.11g as illustrates in Fig.17.

\subsection{NS2 Parameters}

There are many variables in NS2 give the user the ability to control and adjust the flow control. An example of such variables is the interval variable which is defined in the construction of a CBR connection. Interval variable is responsible for the determination of the intervals between sending the packets. Different values of this variable provide different throughput performances. Small intervals lead to different in the throughput performances under the three standards as illustrates in Fig.18 in despite of big intervals 


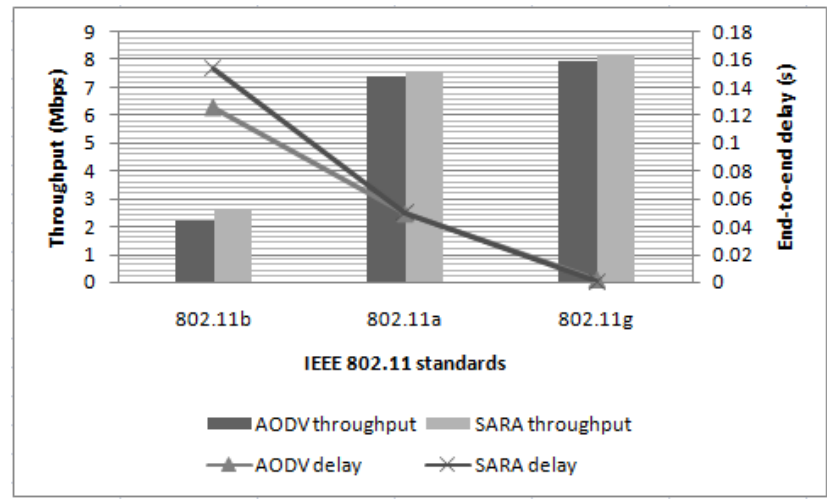

Fig. 15. Parallel - First topology performances

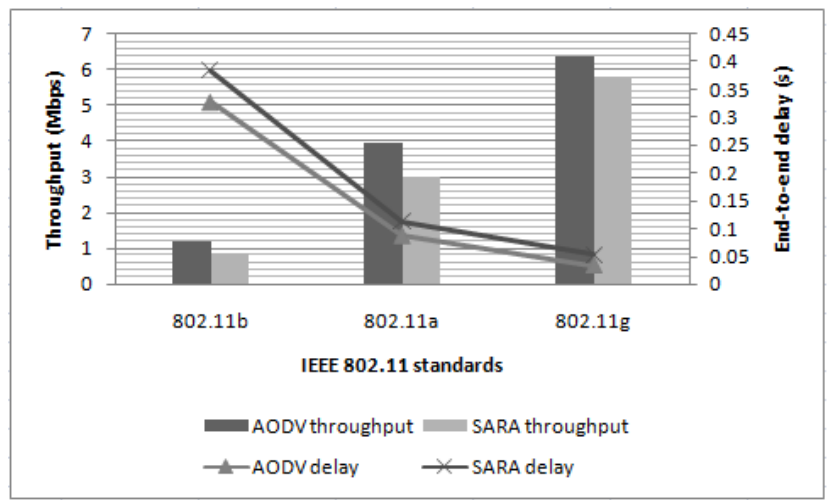

Fig. 16. Parallel - Second topology performances

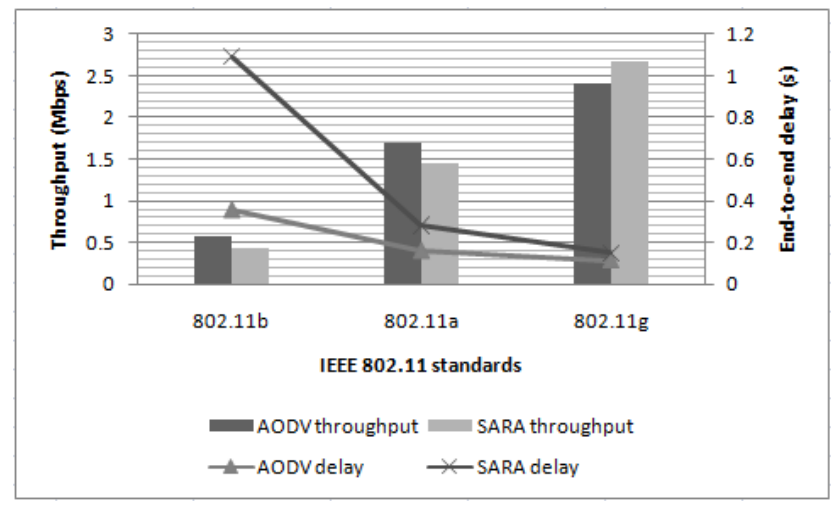

Fig. 17. Parallel - Third topology performances

which lead to the same throughput performance behavior under the three standards as illustrates in Fig.19, the performance of SARA algorithm has the same behavior under the three standards.

\section{CONCLUSION}

In this paper, an empirical study of the elements that may affect a routing algorithm's performance using NS2 simulator is presented. The candidate elements were found to be: different interference lev-

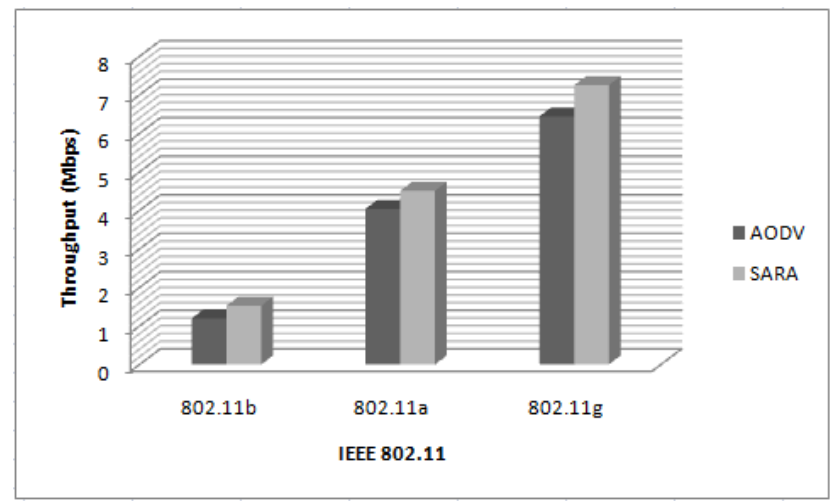

Fig. 18. Small interval performance

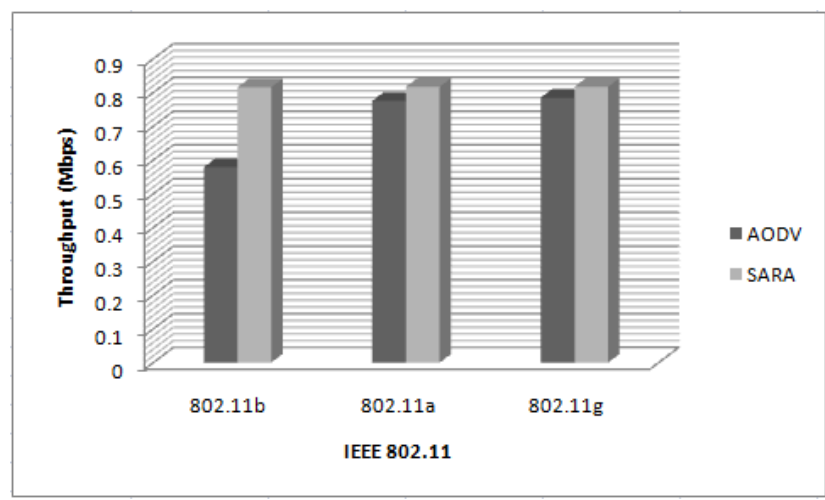

Fig. 19. Big interval performance

els, IEEE 802.11 specifications and data rates. The study has been applied on AODV and SARA routing algorithms. Their performances have been investigated under different scenarios. NS2 has been used to simulate three IEEE 802.11 standards. NS2 has been used to simulate three topologies then has been used to measure their metrics' performances. The performance metrics that have been used are average throughput and end-to-end delay.

The results show that in general the throughput performance behavior of the two routing algorithms is so close to each other under the three standards. AODV routing algorithm performs better in low interference scenarios and SARA routing algorithm performs better in high interference scenarios. The end-to-end delay decreases while the data rate increases in despite of the average throughput which may not proportionally increase with the data rate increase. The future work will include simulations for more routing algorithms and IEEE 802.11 standards. Also, it will include a real test bed environment to execute the simulated results in a real situation.

\section{REFERENCES}

[1] S.K. Sarkar, T.G. Basavaraju and C. Puttamadappa, " Ad hoc mobile wireless networks: principles, protocols and applications," CRC Press, 2007.

[2] E. Osagie, P. Thulasiraman and R.K. Thulasiram, "PACONET: imProved ant colony optimization routing algorithm for mobile ad hoc networks,"Advanced Information Networking and Ap- 
plications, 2008. AINA 2008. 22nd International Conference on. IEEE, pp. 204-211, 2008.

[3] C.E. Perkins and P. Bhagwat, "Highly dynamic destinationsequenced distance-vector routing (DSDV) for mobile computers,"ACM SIGCOMM computer communication review, Vol. 24, No. 4, pp. 234-244, Oct. 1994.

[4] T. Clausen and P. Jacquet, "Optimized link state routing protocol (OLSR)," No. RFC 3626, 2003.

[5] C. Perkins, E. Belding-Royer and S. Das, "Ad hoc on-demand distance vector (AODV) routing," No. RFC 3561. 2003.

[6] D. Johnson, Y. Hu and D. Maltz, "The dynamic source routing protocol (DSR) for mobile ad hoc networks," No. RFC 4728. 2007.

[7] S.R. Das, R. Castaneda, J. Yan and R. Sengupta, "Comparative performance evaluation of routing protocols for mobile, ad hoc networks," Computer Communications and Networks, 1998. Proceedings.7th International Conference on. IEEE, pp. 153-161, Oct. 1998.

[8] M. Günes, M. Kähmer and I. Bouazizi, ”Ant-routing-algorithm (ARA) for mobile multi-hop ad-hoc networks-new features and results," the second mediterranean workshop on ad-hoc networks, Jun. 2003.

[9] F. Correia and T. Vazâo, "Simple ant routing algorithm strategies for a (Multipurpose) MANET model," Ad Hoc Networks, 8(8), pp. 810-823, 2010.

[10] F.A. Bernat, "Simulation of Ant Routing Protocol for Ad-hoc networks in NS-2,"Faculty of Electrical Engineering, Mathematics and Computer Science Network Architectures and Services Group, Nov. 2006.

[11] M. Skariah and C.D. Suriyakala, "An Analysis on the Performance Evaluation of Routing Protocols in Wi-Fi/802.11 b Network," 2014.

[12] F.D. Sanchez Vizcaino and C. Hernandez Benet, "Study of TCP Available Bandwidth Using NS2 and Its Forecasting Based on Genetic Algorithm," 2014.

[13] M. Dorigo, M. Birattari and T. Stutzle, "Ant colony optimization-artificial ants as a computational intelligence technique,"IEEE Computational Intelligence Magazine 1, no. 4, pp.28-39, Nov. 2006.

[14] G. Di Caro, F. Ducatelle and L.M. Gambardella, "Swarm intelligence for routing in mobile ad hoc networks," IEEE Swarm Intelligence Symposium, pp. 76-83, Jun. 2005.

[15] I. Enchev Pervasive, "MANET routing protocols based on swarm intelligence", 2011.

[16] M. Manjunath and D.H. Manjaiah, "PAR: PETAL ANT ROUTING ALGORITHM FOR MOBILE AD HOC NETWORK," International Journal of Computer Networks and Communications, 7(2), p.45, Mar. 2015.

[17] D. Kaur and N. Kumar, "Comparative Analysis of AODV, OLSR, TORA, DSR and DSDV Routing Protocols in Mobile Ad-Hoc Networks,"International Journal of Computer Network and Information Security, 5(3), p.39, Mar. 2013.

[18] T. Bhatia and A.K. Verma, "QoS Comparison of MANET Routing Protocols,"International Journal of Computer Network and Information Security (IJCNIS), 7(9), p.64, Aug. 2015.

[19] F. Correia, Avaialbe:http://cnm.tagus.inescid.pt/software/simple-ant-routing-algorithm-sara, 2012. 